\title{
HOCHSCHULEN
}

\section{Sozialmanagement studieren}

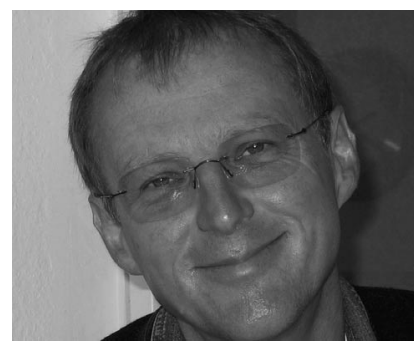

VON PROF. DR. LUDGER KOLHOFF Kolhoff lehrt Soziales Management an der Fakultät Sozialwesen der Ostfalia Hochschule für angewandte Wissenschaften (FH Braunschweig/Wolfenbüttel) Dort leitet er auch den ersten in Deutschland akkreditierten und reakreditierten Studiengang zum "Master of Social Management «. Er ist Mitherausgeber der Schriftenreihen

"Sozialmanagement Praxis" und "Sozialwirtschaft Diskurs" im

Ziel-Verlag Augsburg.

L.Kolhoff@Ostfalia.de
Die Veränderung in der sozialen Landschaft erfordert bei den leistungserbringenden Organisationen mehr und mehr anders orientierte und qualifizierte Führungskräfte: Nicht mehr das an das öffentliche Modell angelehnte Verwaltungshandeln wird verlangt, sondern ein unternehmerisches Management wie in der "freien Wirtschaft". Auf diese Veränderungen haben sich viele Hochschulen nach anfänglichem Zögern - inzwischen eingestellt.

Seit Mitte der 1990er-Jahre werden in der Bundesrepublik Deutschland im sozialen Bereich Marktmechanismen eingeführt: Ausschreibungen von Leistungen, Einführung des neuen Steuerungsmodells, Leistungsverträge, Wechsel von der Sachzur Geldleistung um einen Käufermarkt zu bilden (Bildungsgutscheine, Persönliches Budget etc.). Es wurde eine Konkurrenz um staatlich gestiftete Zahlungsfähigkeit inszeniert (Beckmann/Otto/Schrödter 2009, 30), um durch Wettbewerb mehr Transparenz und in der Folge Kostenreduzierungen zu erreichen. Die vorherrschenden korporatistischen Strukturen wurden durch konkurrenz-, kosten- und leistungsorientierte Auftragsmittelvergabe und die Öffnung für private und gewerbliche Anbieter verändert (Galuske 2007, 345).

\section{Reaktionen der Hochschulen}

Da Konkurrenz und Wettbewerb zunehmend die Sozialwirtschaft bestimmen, benötigt die Praxis pragmatisch orientierte Führungskräfte, die über andere als für das frühere Verwaltungshandeln erforderliche Eigenschaften verfügen (Wöhrle 2008, 21). Benötigt werden Manager, die sich auf der Makro- und Mesoebene der Wohlfahrtsproduktion in politischen Systemen bewegen, um Ressourcen kämpfen und auf Veränderungen der äußeren Rahmenbedingungen flexibel reagieren und ihre Organisationen so aufstellen, dass sie sich im neu entstehenden Wettbewerb behaupten können. Die Initiative, Sozialmanagement-Studiengänge einzurichten, ging deshalb von der Praxis aus und nicht etwa aufgrund von theoretischen Überlegungen innerhalb der Hochschulen, die die Bedeutung des Managements für die Weiterentwicklung der Sozialen Arbeit erkannt hätten (Klüsche 2004).

Doch auf diesen Bedarf wurde an den Fachbereichen Sozialwesen nur zögerlich reagiert. Einerseits waren die bestehenden Strukturen eher verstehend hermeneutisch geprägt, während das Sozialmanagement eine pragmatische Orientierung verlangt; andererseits wurde Sozialmanagement als Versuch verstanden, das Feld der Sozialen Arbeit auf einen neu entstehenden Sozialmarkt hin auszurichten und zu rationalisieren. Befürchtet wurden Standardisierungen und Taylorisierungen, die eine Dequalifizierung der Sozialen Arbeit nach sich ziehe (Beckmann/Otto/Schrödter, 2009, 30).

Auch haftete Leitungsfunktionen ein negatives Image an, denn betriebswirtschaftliche Rationalitäten spielten bisher in der Produktion sozialer Dienstleistungen eine untergeordnete Rolle. Im funktionalen Dilettantismus des »Dritten Sektors « (Seibel 1994), kam man nur mit »Stallgeruch « an die Spitze (Wöhrle 2008, 15).

Also gab es in der zweiten Hälfte der neunziger Jahre neben einigen wenige Weiterbildungsangeboten, nur zwei grundständige Studiengänge an der Berufsakademie in Villingen-Schwenningen und der Fachhochschule Mönchengladbach und universitäre Sozialmanagement-Studiengänge existierten im deutschsprachigen Raum in Österreich (in Wien und Linz) und in der Schweiz an der Universität Freiburg.

Wolf Rainer Wendt, der 1998 als Vorsitzender der Deutschen Gesellschaft für Sozialarbeit zu einer Tagung nach Frankfurt am Main eingeladen hatte, um den Stand der Ausbildung im Sozialmanagement und in der Sozialwirtschaft zu diskutieren, skizzierte folglich ein noch diffuses Bild. Für ihn verknüpften die für die Sozialwirtschaft angebotenen Ausbildungen in unterschiedlicher Weise die inhaltlich von der Managementtheorie der Organisations- und Verwaltungslehre, der Betriebswirtschaftslehre und der Sozialarbeitswissenschaft herkommenden Stränge. Er beobachtete unklare Abgrenzungen und Zuordnungen im Wissensgebiet sozialwirtschaftlicher Praxis, die es 
anderen Fachgebieten erlaubten, das Gebiet der Sozialwirtschaft mit einzubeziehen, sei es der Bereich des Public Managements oder das Feld der pflege- und gesundheitsberuflichen Ausbildungsgänge (Wendt 1999, 85).

\section{Wachstum der \\ Sozialmanagementstudiengänge}

Zwar hat sich an diesem diffusen Bild in den vergangenen zehn Jahren wenig geändert, doch war in der Dekade ein starkes Wachstum an Sozialmanagement-Studiengängen zu verzeichnen, so dass es mittlerweile kaum noch eine einschlägige (Fach-) Hochschule mehr gibt, die nicht über ein Studienangebot zum Sozialmanagement verfügt (Möller 2007, 377).

Der größte Teil der Studienangebote (zwei Drittel) findet an Fachhochschulen statt. Angesiedelt sind die Studienangebote zu 50 Prozent an Fachbereichen Sozialwesen, zu 25 Prozent an wirtschaftswissenschaftlichen und zu zehn Prozent an theologischen Fakultäten oder Fachbereichen. Der Rest verteilt sich auf juristische und verwaltungswissenschaftliche Disziplinen (Boeßenecker/Markert 2007, 33 f.). Sozialmanagement wird also vorwiegend von Fakultäten der Sozialarbeit und Sozialpädagogik angeboten und entwickelte sich in Deutschland aus der Fachlichkeit der Sozialen Arbeit heraus, mit betriebswirtschaftlichen Ansätzen ergänzt, um für Leitungs- und Führungspositionen in der Sozialwirtschaft zu qualifizieren.

Der Anteil der grundständigen Studiengänge beläuft sich auf 30 Prozent und der der postgradualen, die oftmals berufsbegleitend als Fernstudium angeboten werden, beträgt mehr als 70 Prozent. Diese Angebote setzen in der Regel ein Studium der Sozialarbeit und Sozialpädagogik voraus und folgen der Überlegung, dass Management-Qualifikationen in der Sozialen Arbeit nur dann sinnvoll zu entwickeln sind, wenn vorher eine berufsfeldbezogene Grundqualifikation in der Sozialen Arbeit erworben wurde und dadurch eine Verbindung von inhaltlichfachlichen Qualifikationen der Sozialen Arbeit mit Qualifikationen des Managements hergestellt wird (Merchel 2009, 14)). Über die Hälfte der Studienangebote sind als Masterprogramm konzipiert (Boeßenecker/ Markert 2007, 35).

\section{Was sich hinter vielen Studiengängen verbirgt}

Doch was verbirgt sich hinter diesen vielen Studiengängen und sind es wirklich so viele Angebote, wenn man Studiengänge beispielsweise der Hochschulen Dresden und Mittweida, die doppelt aufgelistet wurden
(Boeßenecker/Markert 2007,48-49), herausrechnet, obwohl sie in Kooperation nur einmal existieren oder Lehrgänge, die teilweise nur über einige Wochen gehen, beispielsweise an der Universität Freiburg Schweiz (Diplom-Lehrgang Fundraising, Verbands-NPO-Management etc.) (Boeßenecker/Markert 2007, 48, 76-79).

Die Zahl scheint etwas zu hoch gegriffen. Auch stellt sich die Frage, was man alles unter der Überschrift »Sozialmanagement studieren « subsumieren kann. Neben Studiengängen, die explizit für das Feld des Sozialmanagements qualifizieren wollen, werden von Boeßenecker/Markert (2007) auch Studiengänge aufgelistet wie »Diakoniewissenschaft « (Kirchliche Hochschule Bielefeld, Universität Heidelberg, Fachbereich Evangelische Theologie) und »Humanitäre Hilfe« (Universität Bochum).

$\mathrm{Nach}$ wie vor gibt es also unklare Abgrenzungen und Zuordnungen. Die Anbieterlandschaft scheint zersplittert zu sein, es gibt keine Einigkeit über die Rahmenbedingungen und Inhalte der Ausbildung, wenig Zusammenarbeit zwischen den Trägern und kaum länderübergreifende Kooperationen. Das Vakuum nutzen Hochschulen in privatwirtschaftlicher Trägerschaft, die zunehmend in den Markt drängen (Boeßenecker/Markert 2008, 89).

\section{Was vermittelt wird}

Sylvia Kempe (vormals Albert) hat 2003 eine auf der Studie von Boeßenecker/Markert aufbauende, vergleichende Analyse von an deutschsprachigen Hochschulen angebotenen Sozialmanagement-Studiengängen durchgeführt (Albert 2003) und gefragt, welches grundlegendes Know-how soziale Unternehmen von den Absolventinnen und Absolventen der Sozialmanagement-Studiengänge erwarten dürfen.

In der Untersuchung hat sich gezeigt, dass die Angebote regional ausgewogen und durch »Vielfalt « gekennzeichnet sind. So gibt es die zahlreichen Studiengangsbezeichnungen und Studienpläne.

Ein den Sozialmanagement-Studiengängen gemeinsames zeitlich definierbares Kerncurriculum ist allerdings statistisch nicht nachweisbar (Albert, 2003). Dies gilt auch für Studienangebote, die ihre Studienpläne im Kontext des Bologna-Prozesses modularisiert haben.

Eine Ausnahme bilden die Studienverbünde, die ein gemeinsames Curriculum jeweils geringfügig modifiziert an verschiedenen Hochschulen unter gleichem Namen und mit gleichem Abschluss anbieten (Kempe, 2009, 94). Insbesondere das vom Fernstudienverbund der Länder entwickelte Angebot ist breit vertreten, sei es in Braunschweig, Berlin, Cottbus, Dresden, Mitt- weida oder München, in modifizierter Form auch an anderen Orten und scheint schon fast strukturprägend zu sein.

\section{Lehrbuchreihen als strukturprägendes Element}

Strukturprägend sind auch die beiden Lehrbuchreihen "Sozialmanagement Praxis ", die von Schwarz, Beck, Kolhoff, Schellberg und Wöhrle im Zielverlag (www.zielverlag.de) herausgegebenen wird und "Sozialmanagement/Sozialwirtschaft in der Ausbildung ", die von Wöhrle im NomosVerlag (www.nomos.de) betreut wird. In vielen Studiengängen werden Publikationen aus diesen Reihen eingesetzt. Sie haben damit eine starke inhaltliche Strukturierungsfunktion. Neben diesen beiden Reihen existieren Lehrbücher von Merchel, Knorr, Müller-Gehrmann, Schubert u. a. in diversen weiteren Verlagen. Auch hier ist das Feld durch Konzentration und Diffusion gekennzeichnet, durch eine Ballung von Lehrbüchern im Ziel-Verlag und Nomos-Verlag sowie weitere vereinzelte Angebote.

\section{Resümee: eine Erfolgsgeschichte}

Zusammenfassend ist zu sagen: Die Geschichte des Sozialmanagements an den Hochschulen ist zu einer Erfolgsgeschichte geworden.

Die Implementierung scheint abgeschlossen zu sein und Sozialmanagement ist aus dem Bereich der Sozialen Arbeit und der Sozialpädagogik nicht mehr wegzudenken. Es zeigt sich, dass der Bereich Sozialmanagement in der Hochschullandschaft fest verankert ist und seinen Platz insbesondere im Fachhochschulbereich in den Fachbereichen für Soziale Arbeit gefunden hat. Doch ist auch ein Verdrängungswettbewerb zu verzeichnen. So konnte eine zunehmende Zahl unterschiedlicher Träger aus finanziellen oder logistischen Gründen ihren Studienbetrieb nicht aufrecht erhalten.

Doch das Bild ist zum Glück nicht einheitlich. Zum einen gibt es neue Netzwerke, zum anderen verfestigen sich bestehende Strukturen. Daneben gibt es weitere Ausdifferenzierungen, beispielsweise in Bereichen wie Senioren-Management, Kita-Management, Behinderten-Management etc.

Literaturverzeichnis nächste Seite 


\section{Literatur}

Albert, S., Vergleichende Analyse von an deutschsprachigen Hochschulen angebotenen Sozialmanagement-Studiengängen mit einem akademischen Abschluss anhand ihrer Curricula, Masterarbeit an der Hochschule Mittweida, Fachbereich Soziale Arbeit, Aufbaustudium Sozialmanagement, Mittweida 2003 (unveröffentlicht). Bassarak, H., Wöhrle, A. (Hg.), Sozialwirtschaft und Sozialmanagement im deutschsprachigen Raum, Bestandsaufnahme und Perspektiven, Augsburg 2008.

Beckmann, Ch., Otto, H.-U, Schrödter, M., Management der Profession: Zwischen Herrschaft und Koordination, in, Grunwald, K. (Hrsg.), Vom Sozialmanagement zum Management des Sozialen? Eine Bestandsaufnahme, Hohengehren 2009, 15-41.

Boeßenecker, K.-H., Markert, A., Sozialmanagement studieren, Studienangebote im Bereich Sozialmanagement und Sozialwirtschaft und Analysen veränderter Rahmenbedingungen. Hans Böckler Stiftung, Arbeitspapier, Nr. 141, Düsseldorf 2007.

Boeßenecker, K.-H., Markert, A., Studiengänge im Bereich des Sozialmanagements in der Sozialwirtschaft - Bestandsaufnahme, Konzepte, Perspektiven, in, Bassarak, H., Wöhrle, A. (Hg.), Sozialwirtschaft und Sozialmanagement im deutschsprachigen Raum, Augsburg 2008, 88-91. Brinkmann, V. (Hg), Personalentwicklung und Personalmanagement in der Sozialwirtschaft, Tagungsband der 2. norddeutschen Sozialwirtschaftsmesse, Wiesbaden 2008.

Galuske, M., "Wenn Soziale Arbeit zum Management wird ...", Anmerkungen zum aktivierenden Umbau der Sozialen Arbeit und seinen Niederschlägen in der Methodendebatte, in, Krauß, E. J., Möller, M, Münchmeier, R. (Hg.), Soziale Arbeit zwischen Ökonomisierung und Selbstbestimmung, Kassel 2007, 333-372. Grunwald, K. (Hg.), Vom Sozialmanagement zum Management des Sozialen? Eine Bestandsaufnahme, Hohengehren 2009.

Kempe, S., Vergleichende Untersuchung der deutschsprachigen Sozialmanagementstudiengänge anhand ihrer Curricula, in, Bassarak, H., Wöhrle, A. (Hg.), Sozialwirtschaft und Sozialmanagement im deutschsprachigen Raum, Bestandsaufnahme und Perspektiven, Augsburg 2008, 92-96.

Klüsche, W., Zur Zukunft des Sozialmanagements, Vortrag auf der Tagung "Qualitätsmanagement von Studiengängen des Sozialmanagements und der Sozialwirtschaft" der Bundesarbeitsgemeinschaft Sozialmanagement an Hochschulen e. V. in Mönchengladbach, Juni 2004 (unveröffentlicht).

Kolhoff, L., Hochschulausbildung im Bereich des Sozialmanagements in der Sozialwirtschaft - Bestandsaufnahme, Konzepte, Perspektiven, in, Bassarak, H., Wöhrle, A. (Hg.), Sozialwirtschaft und Sozialmanagement im deutschsprachigen Raum, Bestandsaufnahme und Perspektiven, Augsburg 2008, 78-87.
Krauß, E. J., Möller, M, Münchmeier, R. (Hg.), Soziale Arbeit zwischen Ökonomisierung und Selbstbestimmung, Kassel 2007.

Merchel, J., Sozialmanagement, Eine Einführung in Hintergründe, Anforderungen und Gestaltungsperspektiven des Managements in Einrichtungen der Sozialen Arbeit, 3. Auflage, Weinheim und München 2009.

Möller, M., Was ist und zu welchem Ende betreiben wir Sozialmanagement? Oder über den Zusammenhang zwischen sozialpolitischer Entwicklung, Sozialmanagement, Prekarisierung der Professionellen und deren Ausbildung in der sozialpädagogischen Arbeit, in, Krauß, E. J., Möller, M, Münchmeier, R. (Hg.), Soziale Arbeit zwischen Ökonomisierung und Selbstbestimmung, Kassel 2007, 377-396.

Seibel, W., Funktionaler Dilettantismus. Erfolgreich scheiternde Organisationen im "Dritten Sektor " zwischen Markt und Staat, 2. Auflage, Baden-Baden 1994.

Wendt, W. R., Sozialwirtschaft und Sozialmanagement in der Ausbildung, Baden-Baden 1999.

Wöhrle, A., Der zweite Professionalisierungsschub durch Sozialmanagement, in, Brinkmann, V. (Hg), Personalentwicklung und Personalmanagement in der Sozialwirtschaft, Tagungsband der 2. norddeutschen Sozialwirtschaftsmesse, Wiesbaden 2008, 13-40.

\section{Veränderungs- prozesse in sozialen \\ Unternehmen}

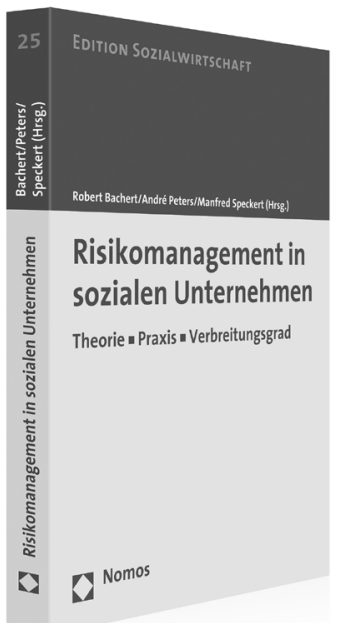

\section{Risikomanagement in sozialen Unternehmen}

Theorie - Praxis -

Verbreitungsgrad

Herausgegeben von Robert Bachert, André Peters und Manfred Speckert

2008, 294 S., brosch., 49,-€, ISBN 978-3-8329-3721-8

(Edition Sozialwirtschaft, Bd. 25)

Finanzierungsengpässe der öffentlichen Hand, demografischer Wandel, neue betriebswirtschaftliche Erkenntnisse: Wie können sich soziale Unternehmen auf Veränderungsprozesse optimal einstellen? Die Autoren erläutern, wie Risikomanagement im Zusammenspiel mit strategischem und operativem Controlling und mit den Grundsätzen von Corporate Governance zielgerichtet entwickelt und eingeführt werden kann. 